\title{
Zum Ausnahmezustand als Experimentierfeld für die Legitimierung von sonder- und außerrechtlichen Maßnahmen
}

\author{
Anna-Lena Dießelmann (Siegen)
}

\begin{abstract}
This study focuses on the protests against the G8 summit in Heilgendamm, Germany, 2007. In the weeks preceding the event, Germany was preparing for a state of emergency. This discourse was created by legal authorities, politicians, security experts, the international media and the protestors alike. The study reconstructs the struggle for legitimacy and hegemonic supremacy. During this process the definition what was considered a "normal" protest and what a transgression shifted - highlighted by the demeanor of the special police task force BAO Kavala, but also by the gradual establishment of emergency laws for the normal case. The BAO expressed their intention to influence the public opinion explicitly.
\end{abstract}

\section{Von Höhen und Tiefen}

Im Zentrum des Artikels steht eine exemplarische Fallstudie des sogenannten "Gipfeltreffens" der damaligen G81 in Heiligendamm im Jahre 2007. Da große Demonstrationen aus dem gesamten Spektrum der GlobalisierungskritikerInnen angekündigt waren und es nicht zuletzt um das Prestige des gastgebenden Landes geht, wurde das Ereignis zum Labor der deutschen Sicherheitsgarantie. Bereits jetzt lässt sich im Hinblick auf den anstehenden G8-Gipfel 2015 in Deutschland ein Diskurs unter absoluter Vormachtstellung des Hochwertbegriffs Sicherheit ${ }^{2}$ beobachten. So titelte z. B. der Merkur bereits am 24.01.2014: "Der G8-Gipfel 2015 findet auf Schloss Elmau statt. In aller Abgeschiedenheit wollen die Mächtigen tagen. Der logistische Aufwand wird enorm - vor allem in puncto Sicherheit". Diese Einschätzung bezieht sich auf eine Analyse des letzten in Deutschland ausgetragenen Gipfeltreffens 2007 in Heiligendamm:

Vom G8-Gipfel 2007 in Heiligendamm sind zwei Bilder im Gedächtnis haften geblieben: Hier die mächtigen Regierungschefs, die sich in einen überdimensionalen Strandkorb quetschten. Dort vermummte Demonstranten, die sich gegen den Strahl der Wasserwerfer stemmen. In Zahlen: 16.000 Polizisten, 1.060 Festnahmen, aber auch 5.000 Journalisten aus 73 Ländern. Das

\footnotetext{
${ }^{1}$ Inzwischen sind es wieder die ehemaligen G7, nachdem die zeitweilige Teilnahme Russlands wegen des Ukrainekonflikts einseitig beendet wurde. Diese Gipfeltreffen finden rotierend in den beteiligten Ländern statt.

${ }^{2}$ Kursivierungen markieren im Folgenden Ausdrücke, die als Topoi im Analysekorpus vorkommen, GROSSBUCHSTABEN dahingegen analytisch gewonnene, übergeordnete Kategorien. 
Ostseebad war einige Tage der Mittelpunkt der Welt. Am Ende kostete MecklenburgVorpommern das Spektakel rund 60 Millionen Euro.

(Schier/Holzapfel/Schnürer 2014)

Die Nachbetrachtung der Ereignisse um den G8-Gipfel 2007 zeigt, welche Bilder sich in der Öffentlichkeit manifestiert haben. Ein Bild, das nach der Analyse der seitens Polizei und Innenministerien konstruierten LAGE- und FEINDBILD-Konstellationen nicht überrascht, auch wenn es verwundert, dass viele Falschmeldungen nach Jahren noch unkorrigiert wiedergegeben werden. ${ }^{3}$ Diese Darstellung des quantitativen Ausmaßes der Proteste diente indirekt zur Rechtfertigung der Polizeistrategie und wurde mit weiteren Daten gerahmt: Ohne die "Erfolgsmeldung" der Festnahmen würden sich auch die eingesetzten PolizistInnen und Millionen Euros nur schwer öffentlich und politisch rechtfertigen lassen.

Während der Arbeit an diesem Artikel werden weitere Staatsausgaben in Milliardenhöhe mit Sicherheit legitimiert: Gegen die angebliche Bedrohung durch Flüchtlinge nach Europa, gegen Terrorismus und erst recht nach dem Mordanschlag auf die Redaktion des Charlie Hebdo. Über die Lage nach dem Anschlag wurde in Medien als "Ausnahmezustand", "Notstand" zuweilen sogar als "Kriegszustand" berichtet. Die Fragestellung der vorliegenden Analyse ist demnach aktuell und relevant, erfordert jedoch auch eine Auseinandersetzung mit den unterschiedlichen "Grauzonen", in denen westliche Demokratie mit den Charakteristika "postdemokratischer" Regime, mit verschiedenen Stufen des "Maßnahmenstaats" (Fraenkel 2012) beschrieben werden kann. Bei allen Nuancen des Ausnahmezustands sind neben materiellen oder dispositiven Elementen wie Polizeikräften und Einsatzabteilungen anderer Behörden oder dem Militär immer diskursive Elemente konstitutiv: Diese gilt es im Folgenden zu zeigen.

Diese Analyse zeigt die Kommunikation staatlicher Akteure auf, die auf besondere Weise an rechtliche Regeln gebunden sind, diese allerdings nachweislich in ihrem Handeln nicht nur überschreiten, sondern den Handlungsrahmen gar transformieren. Das Ziel einer solchen Analyse ist es, einen Zusammenhang zwischen rechtlicher Normgenese und mediopolitischem Interdiskurs nachzuweisen - also die Transkriptionskette interner Sprachmuster auf Kommunikation nach außen und schließlich deren Manifestation in Rechtsetzung und Rechtsprechung. Ausgehend von der These, dass Normen und Ausnahmen gesellschaftlich erzeugte, zentrale Kategorien sind, die immer im Kontext von Macht und Ausschluss stehen, soll in diesem Beitrag analysiert werden, wie über den Rekurs auf die Ausnahme Normalität (re)konstruiert wird (vgl. Schmitt 1932/1996a). Mit anderen Worten: Es wird untersucht, wie der permanente Ausnahmezustand zum Dispositiv des Regierens wird. Obwohl das Ereignis Heiligendamm zunächst ein begrenzter Ausnahmezustand ist, lässt sich daran zeigen, wie sonder- und extrarechtliche Zustände für den Alltag normalisiert werden.

Als Nachweis der diskursiven Konstitution von Legitimität eigenen Handelns dienen auf erster Ebene Aufzeichnungen verschiedener Polizei-interner Kommunikationsvorgänge. Dazu

\footnotetext{
${ }^{3}$ So hatten z. B. nur drei dieser angeblichen 1.060 Festnahmen - es handelt sich um Ingewahrsamnahmen - zu Verurteilungen geführt. Zahlen als Verdatungen wurden öffentlich wirksam als Legitimationsressource verwendet, denn solche Ziffern und Statistiken wirken in einer normalistischen Gesellschaft wie neutrale Belege (vgl. Link 2009).
} 
gehören u. a. Protokolle von Telefonaten, vom internen Radio während des Einsatzzeitraums und von Arbeitstreffen, interne Mitteilungen, Notizen, Einsatzhandbücher und Anweisungen ${ }^{4}$. Auf zweiter Ebene wird an einigen Beispielen die Übernahme dieser polizeilichen Kommunikation - insbesondere der Personen- und Lagebeschreibungen - in Gerichtsurteilen nachgezeichnet. Somit wird der Einfluss der Exekutive auf die Judikative nachgewiesen. Auf dritter Ebene dokumentieren u. a. die Pressemeldungen, öffentliche Reden und Mitteilungen an die Bevölkerung von der Polizei und der polizeilichen Sonderbehörde Kavala ${ }^{5}$ die Umsetzung der intern verhandelten Kommunikationsstrategie mit der Öffentlichkeit. Daran werden die Motive und Strategien der polizeilichen Kommunikation untersucht. Denn Intention jeder (polizeilichen) Öffentlichkeitsarbeit ist die Hegemonialisierung von Deutungsmustern zur Legitimation eigenen Handelns. Die Analyse dieser Diskursposition im Sicherheits- und Krisendiskurs ${ }^{6}$ und ihres Zusammenhangs im diskursiven Gefüge ermöglicht die Aufdeckung der Strategien zur Übernahme konkreter, rechtsbindender Bezeichnungen aus "Ausnahmezuständen" in die "Normalität". Das Politische und das Mediale sind untrennbar miteinander verflochten, in diesem Sinne und im Anschluss an Link (1992) und Jäger und Jäger (2007) wird davon ausgegangen, dass im mediopolitischen Diskurs Normen und Legitimität permanent verhandelt. Der Zusammenhang von Legitimität und Normalität kann besonders an Situationen des Ausnahmezustands aufgezeigt werden, in denen und mittels derer Handlungen entgegen der gegebenen Legalität implementiert werden und daher besonderer Begründung bedürfen. Hegemonie entsteht in zeitlichem Abstand, durch langwierige Prozesse der Implementierung von Legitimität, die erst in der Rückschau abschließend bewertet werden können. In dieser exemplarischen Studie stehen besonders diejenigen Bedingungen auf dem Prüfstand, die nicht zuletzt zur Änderung von Gesetzen und deren Anwendung geführt haben und somit bestehende Legitimitätsordnungen modifizieren konnten und weiter verändern werden.

\footnotetext{
${ }^{4}$ Das Material für diese Untersuchung der internen Kommunikationswege wurde zum größten Teil im Zentralarchiv der Polizei in Mecklenburg-Vorpommern erhoben.

${ }^{5}$ Die Besondere Aufbauorganisation (BAO) Kavala wurde bereits lange im Vorfeld eigens gegründet und mit der gesamten Planung und Durchführung des Gipfels betraut. Die BAO arbeitete mit Verbindungsbeamten aus Bundeswehr und Feuerwehr und hatte ihren Sitz in Mecklenburg-Vorpommern.

${ }^{6}$ Analytische Arbeiten zur Geschichte des Sicherheitsdiskurses setzen in den 1970er Jahren an (vgl. Galli/Preußer 2006) und registrieren eine Verschiebung der Bedrohungsszenarien: Zuvor bezog sich die Konstruktion der Bedrohung auf ein bestimmtes Phänomen oder eine spezifische Gruppe wie z. B. Dealer, Gewalttäter oder Migranten als Gefahrenquelle (vgl. Link 2006; Köster 2009), gegenwärtig auf eine generelle potenzielle Gefahr. Nach der Verstaatlichung der letzten Stadtpolizei 1975 in München verschwand das Thema "Sicherheit" zunächst aus der kommunalpolitischen Diskussion (vgl. Eick/Töpfer 2007). "Gefährliche Orte" und "Angsträume" sind seit den 1990er Jahren wieder entdeckt und besonders in den Diskurs über "Städte" indiziert worden. Diese subjektiven Gefühle werden ent-subjektiviert und dienen zur Legitimation von neuen Repressionstechniken wie im Zusammenhang mit der Terrorismusbekämpfung seit 9/11 (vgl. Link 2001). Der Terrorismus wird zur permanenten Bedrohung stilisiert und somit die Lage als im permanenten Ausnahmezustand beschrieben (vgl. Agamben 2004). Aus dem Sicherheitsdiskurs können politische Handlungen abgeleitet werden: 2002 hat die diskursive Propagierung der Gefahr zum Erlass des Gesetzes zur Bekämpfung des internationalen Terrorismus geführt. Zusätzlich bestimmen Kriegsmetaphern wie "war on terror" den aktuellen Elementardiskurs, so dass zivile Abwehrmaßnahmen mit militärischen Konnotationen durchsetzt werden.
} 


\section{Zum Verständnis von Norm und Ausnahme}

Wie Spindler und Tonks (2007: 5) feststellen, orientiert sich

"Normalität" an wechselnden Sagbarkeitsfeldern innerhalb einer Gesellschaft und differiert von Kultur zu Kultur. [...] Dennoch lässt sich eine gewisse common sense-Definition von Normalität aufspüren, die vor allem dann zum Tragen kommt, wenn ein gesellschaftlicher Zustand als "nicht normal", als Ausnahmezustand, empfunden wird.

(Spindler/Tonks 2007: 5; Hervorhebung im Original)

Unzählige Beispiele und wiederkehrende Strategien zeigen die Relevanz einer übergreifenden Analyse der Funktion dieses Ausnahmezustands in gegenwärtigen Diskursen ${ }^{7}$ : Die Finanzkrise wird als Ausnahmesituation beschrieben und so der Sparzwang legitimiert (vgl. Parr 2010; Müller/Schmitz 2010), Bundeswehreinsätze im Innern werden mit Ausnahmezuständen begründet (vgl. Rucht/Teune 2008), die angebliche Gefahr durch Terrorismus führt zum alltäglichen politischen Ausnahmezustand (vgl. Galli et al. 2006). Der Ausnahmezustand kann nicht nur als militärischer Notstand ausgerufen werden, vielmehr gehört der sozialpolitische Notstand zum Alltag (vgl. Spindler/Tonks 2007).

Ausnahmerechte und Notstandsrechte, die im Ursprung im strengen Kontrast zur Normalität stehen sollten, sind gegenwärtig normaler Bestandteil des demokratischen Gouvernements. Der Ausnahmezustand ist ein asymmetrischer Zustand. Ein Ausnahmezustand ist kein Gegenstand, den man anschauen oder anfassen kann, sondern ein erzeugtes Bild, das über Sprache evoziert wird. Es entsteht in bestimmten Verhältnissen verschiedener Elemente des Diskurses, sogenannten ratios. Unter ratio verstehe ich nach Burke (1969a: 13) das Verhältnis zwischen den Elementen des Diskurses. In der scene-act-ratio z. B. beinhaltet die Szene den Akt. Typische Argumentationen und Motive wären die Aussagen, die Handelnde zu "prisioners of the situation" machen. Scene-agent-ratios, in denen also Szene und AkteurIn in einer synekdotischen Beziehung stehen, seien nicht einfach durch "containerte" und "containernde" Elemente zu beschreiben, sondern durch ein Zusammenspiel der Personen und des Ortes. Burke folgend wird angenommen, dass wenn Sprache benutzt wird, diese auch die/den SprecherIn benutzt. Krisenrhetorik ist zudem grundlegend geprägt von Dramatisierungs- und parallelen Entdramatisierungsstrategien, die ebenfalls als ratios $^{8}$ analysiert werden. Rolle und Funktion von

\footnotetext{
${ }^{7}$ Unter Diskurs wird in Anlehnung an Foucault ein "Fluss durch Zeit und Raum" verstanden, ein sprachlich produzierter Bedeutungszusammenhang, eine Praxis, die Machtstrukturen als Grundlage hat und diese zugleich erzeugt. Äußerungen werden als nicht wiederholbare, "zeitlich-räumlich spezifische Aussagenereignisse" verstanden und als die Atome des Diskurses analysiert (Bührmann/Schneider 2008: 26). Der Diskurs kann nach Link in drei Diskurstypen - Spezial-, Inter- und Elementardiskurs - unterteilt werden. Diskurslinguistik ist auf Sprache fokussiert, integriert $\mathrm{Ko}(\mathrm{n})$ texte in die Interpretation und ermöglicht so einen prinzipiellen Zugang zu den Textnetzen der internen und öffentlichen Kommunikation und des Rechts. Einzelne Texte verlieren in der Analyse ihren individuellen Status und werden verstanden als konstitutiver Teil z. B. juristischer Wissensarchitekturen. Diese Methoden der Diskursanalyse eignen sich dazu, verschiedene Typen und Stränge sowie Topoi aufeinander beziehen zu können und vergleichbar zu machen, um Deutungsrahmen und Möglichkeiten des Wissens offen zu legen. Mit der Erforschung der Rolle der Medien für den Normalismus lässt sich die "Ausnahme" als mediopolitisches Narrativ verdeutlichen (vgl. Reisigl 2007).

${ }^{8}$ Zunächst macht Burke fünf elementare Einheiten in Szenen allgemein aus. Act als die Handlung selbst; scene als die Situation der Handlung, konkrete Verankerung in Raum und Zeit; agent als die beteiligten Personen, also auch counter-agent, co-agent; agency für die Art und Weise der Handlungsausführung; purpose für Motivation, Intention, Gründe für die Handlung. Grundlegend für Burkes Analysewerkzeug ist die Unterscheidung von
}

ISSN 1615-3014 
Handlungen und Geschehen können in Hinblick darauf untersucht werden, ob sie einen Inhalt transportieren, auslösen, beinhalten oder in einem anderen Terminus beinhaltet sind (vgl. Burke 1969a: 503-504; cf. Abbildung 1).

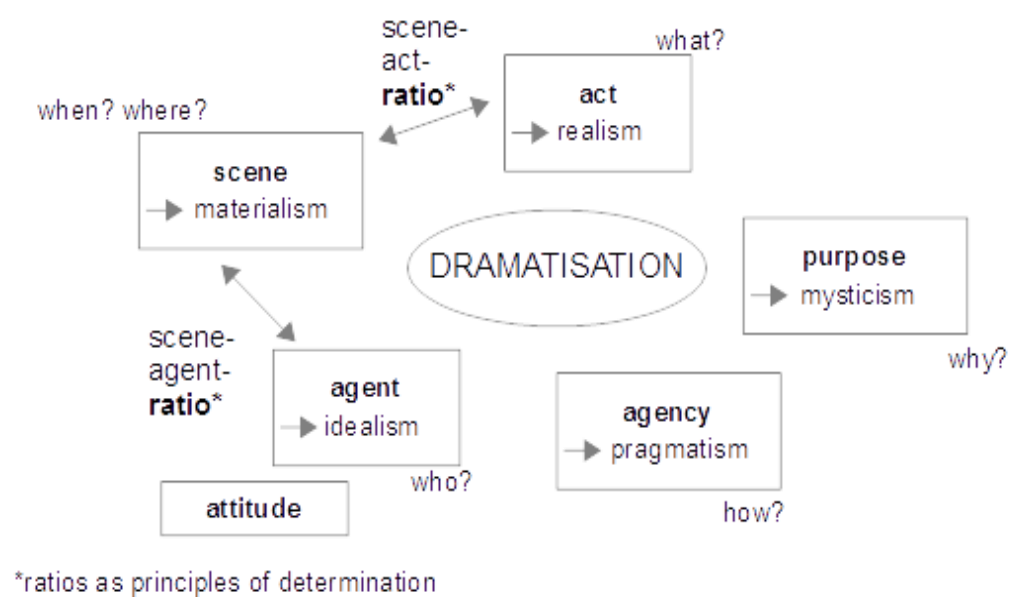

Abbildung 1: Ratios nach Burke. Quelle: Eigene Darstellung

Diese Beschreibung liefert eine grundlegende Möglichkeit, die kausalen - und auch die nicht kausal-logischen - Implikationen und Folgerungen ableiten und nachvollziehen zu können (vgl. Burke 1996a). Die möglichen ratios verstehe ich wie Burke als Prinzipien der Determination von Inhalten. Kennzeichnend für die Krisenrhetorik ist die von Burke für die ratios beschriebene Zuständigkeit sowohl der AkteurInnen als auch der Szene für die Dramatisierung und Schilderung von Ausweglosigkeit. Dabei würden sich viele Argumente und Begründungen weniger auf einzelne Elemente des Diskurses beziehen, sondern auf bestimmte Verhältnisse zwischen diesen Elementen. Burke entwickelte dazu eine Interpretationsweise, die Kommunikation als Drama versteht. Diese Methode lässt auch soziale Beziehungen verstehen, indem sie Gesellschaft mit Drama vergleicht. Durch das Burkesche Modell des dramatism lässt sich erklären, wie Handlungen legitimiert und begründet werden. Der Sachzwanglogik zufolge legitimiert die Krise die Prävention und lässt Prävention sogar alternativlos erscheinen (vgl. Bröckling 2008: 47).

Der Ausnahmezustand als terminus technicus bezeichnet hier ein komplexes Ensemble politischer und rechtlicher Phänomene. Bewusst wird er in Abgrenzung zu den konkreten Bezeichnungen von einzelnen - juristisch mehr oder weniger klar definierten - Sonderrechten wie Notstand, Kriegszustand oder Gefahrensituation analysiert. Denn der Ausnahmezustand wird nicht verstanden als ein einzelnes Sonderrecht, sondern als eine diskursive Strategie. Im Sinne Agambens (2004: 45) wird damit die Markierung der Schwelle zum gemeinhin als Normalität

scene-act-ratio und scene-agent-ratio, also die Unterscheidung von Situationen, in denen die Szene das Handeln bestimmt (scene-act-ratio) und in denen die Szene und die Akteur/innen in einer synekdotischen Beziehung stehen (scene-agent-ratio). Mit diesen Schlüsselbegriffen (key-terms) lässt sich die Dramatisierung von Handlungen und Geschehen nach Burke analysieren, indem ihre Rolle und Funktion untersucht wird. Über die Beschäftigung mit literarischen Texten nähert sich Burke ebenfalls rechtlichen Texten, denn sie enthalten "forms of art", "forms of prophecy and prediction" und ein "level of generalisations that justifies predictions". Mit diesem Grundwortschatz als Werkzeugkoffer lassen sich prototypische politische und juristische Topoi erklären und beschreiben (vgl. Burke 1969a: 503-504). Besonders für Proteste lässt sich die Analysemethode Burkes verwenden, da sie szenische Momente sind. 
Verstandenen verhandelt. Für diese Arbeit von Belang sind daher diejenigen Situationen, die als Ausnahmezustand benannt und dadurch als eine Gefahrensituation konstituiert werden. Denn seit der Einführung der Notstandsgesetze in der BRD wird der Ausnahmezustand nicht formal als Notstand ausgerufen. Stattdessen werden Notstandsklauseln schleichend implementiert, um den Ausnahmezustand für den Normalbetrieb handhabbar zu machen. Die repräsentative Demokratie macht die Legitimation dieser Implementierung obligat. Anhand dieser notwendigen Begründungsmuster lassen sich die sprachlichen Verfahren der Regierungsorgane zur Verschärfung der nicht-demokratischen Praxen nachweisen. Subtil aber alltäglich also normale Praxis - ist die schleichende, durch die Produktion von Feindbildern und Krisensituationen legitimierte Implementierung von Notstandsklauseln. ${ }^{9}$

Urteile werden gesprochen. Gesetze werden verabschiedet, damit sie in Kraft treten. Sie haben in repräsentativen Demokratien keine Geltung ohne den performativen Akt ihrer Versprachlichung. Sowohl Rechtsetzung als auch Rechtsprechung sind sprachliche performative Phänomene. Denn rechtsrelevante LAGEbeschreibungen sind Wissensrahmen, ${ }^{10}$ sie sind verhandelt und stehen stets zur Disposition. Dabei sind die institutionellen Rahmen der Veränderlichkeit stets mehr oder weniger eindeutig vorgegeben. Das zugrundeliegende Verständnis von Normen leitet sich aus den Annahmen des 1) rechtslinguistischen Normbegriffs und des 2) rechtsphilosophischen Normkonzepts ab: Rechtliche Normen sind eine bestimmte Kategorie sozialer Normen, die statt gesamtgesellschaftlich eher innerhalb spezifischer Institutionen verhandelt werden. Rechte sind vorläufige Ergebnisse komplexer sozialer Handlungen. Rechtstexte sind demnach nicht durch übergeordnete Werte bestimmt oder aus ihnen ableitbar (keine Container), sondern sind im Verfassungsstaat Bezugspunkt von Norm-Zuschreibungsdiskursen, dementsprechend "produzieren" sie performativ eben diese Normen und Werte, die sie zugleich beinhalten. Da ihre Anwendung ihre Wirkung erzeugt, ist Rechtsprechung ein performativer Akt der Rechtsetzung. Denn wer Recht spricht, manifestiert Normen und setzt Handlungsrahmen, die als rechtliche Bedingungen gelten können (vgl. Busse 2013). Diese eigene Form der Performativität von Rechtsarbeit kann am Beispiel der polizeilichen Gefahrenbegriffe verdeutlicht werden: Denn die Beschreibung einer Situation als "besondere Lage", "polizeiliche Großlage", "Krise" oder "Ausnahmezustand" ist nicht richtig oder falsch, sondern kann als performative Handlung gelingen oder nicht. Rechtsetzung und Rechtsprechung liegen demnach näher beisammen als weithin angenommen.

\footnotetext{
${ }^{9}$ Hier wird zwischen "normalen Feinden" und dem Abstraktum "Terrorismus" unterschieden, denn "Es geht weniger um den absoluten Feind, der als 'globaler Terrorismus' usw. markiert wird, vielmehr um die alltägliche und 'normale', um die unterschwellige Feindbildproduktion unter den gegebenen Bedingungen, die 'Sicherheit' als letzten Leitwert staatlichen Handelns ausweisen, und 'Prävention' als den Weg dorthin" (Knobloch 2008: 3).

${ }^{10}$ An dieser Stelle sei darauf hingewiesen, dass unter frames oder Wissensrahmen ein mentales Konzept verstanden wird, welches zum Verstehen von sprachlichen Handlungen beiträgt und andere Semantiken um eine notwendige Ebene ergänzt. Das nicht offensichtliche, in gängigen semantischen Beschreibungen und linguistischen Theorien nicht berücksichtigte frame-Wissen greift auf allen Ebenen der Organisation von Sprache ein. Frame-Semantik dient daher auch zur Erfassung und Beschreibung desjenigen Teils von verstehensrelevantem Wissen, das in üblichen semantischen Beschreibungen und Theorien nicht im Fokus steht.
} 


\subsection{Scene: Die Lagebeschreibungen}

Öffentliche wie rechtliche Legitimität polizeilicher Maßnahmen hängt von ihrer jeweiligen Angemessenheit ab. Angemessenheit wird wiederum in Bezug auf zu erwartende Ausnahmezustände bewertet, anhand von Prognosen über die zu erwartende Situation, z. B. die Härte der Auseinandersetzungen. Die vorhergehende Beschreibung einer zukünftigen Lage begrenzt also bereits die möglichen Bewertungen der Angemessenheit. Diese Bewertung der Lage wurde zum G8-Gipfel in Heiligendamm von polizeilichen Stellen selbst vorgenommen.

In der folgenden Tabelle sind solche Begriffe aufgelistet, die zur Beschreibung der LAGE verwendet wurden. Sie stehen teils im Konflikt oder Wettstreit zueinander. Vor allem bilden sie vorläufig das Spannungsfeld ab, auf dem die Strategien der Kommunikation stattfanden angefangen von Beschwichtigungsstrategien bis hin zur diskursiven Konstitution eines Ausnahmezustands. Obwohl sie juristische Konsequenzen haben, sind bei weitem nicht alle Begriffe rechtlich klar definierte Termini.

\begin{tabular}{|l|l|l|l|l|}
\hline & besondere Lage & Gefahr & & Ausnahmezustand \\
Risiko(lage) & $\begin{array}{l}\text { Anscheinsgefahr } \\
\text { polizeiliche } \\
\text { Großlage }\end{array}$ & Gefahrenverdacht & Krise & Notstand \\
& Gefahr im Verzug & & Katastrophe \\
\hline
\end{tabular}

Abbildung 2: Polizeiliche Begriffe der Beschreibung der LAGE. Quelle: Eigene Darstellung

Der sich eröffnende Möglichkeits- oder Handlungsspielraum dieser Begriffe weitet sich von links nach rechts. Diese Begriffe gehören zum kontinuierlichen Repertoire des gesellschaftlichen Möglichkeitsrahmens und machen deswegen ständig Logiken des Vorbeugens glaubhaft. Allen Lagebegriffen ist gemein, dass sie eine Handlungsnotwenigkeit erzeugen. Sie unterscheiden sich jedoch hinsichtlich der Frage, welche Eingriffe legitimiert werden können.

Die unterschiedlichen Lagebeschreibungen implementieren unterschiedliche, zustimmungspflichtige Hochwerte. In der ausführlichen Analyse (vgl. Dießelmann 2015) konnte belegt werden, was hier nur kursorisch angemerkt wird: Das Vorhandensein eines Risikos macht einen Eingriff zur Wiederherstellung der Sicherheit legitim und Risiko fordert vor allem Prävention. Ein Risiko ist im Kontext von Gesetzen anders zu interpretieren als in der Alltagssprache. Ein hohes Risiko würde im alltagssprachlichen Rahmen etwas sein, das es zu verhindern oder zumindest nicht einzugehen gilt, meint aber rechtlich gesprochen etwas nicht Vermeidbares. Die LAGE als Bedrohung oder Gefahr weitet die Legitimierungsmöglichkeiten der Gouvernementalität scheinbar flexibel aus. Dies vor allem, da die Bedeutungen rechtlicher Begriffe nicht aus dem direkten Umfeld im Text gelesen werden können, sondern sich aus weitreichenden impliziten und expliziten Verweisen und intertextuellen Bezügen ergeben. 
Gefahr und Notstand evozieren alltagssprachliche Interpretationen und Verständnisse, die in der rechtskontextuellen Verwendung nicht vergleichbar sind, allerdings durch den Interdiskurs transportiert werden. Der über diese Konstituierungen der Situation induzierte Hochwert Sicherheit kann als agency gelesen und verstanden werden. Deren Skopus war im Krisendiskurs vor 9/11 vorwiegend auf den agent, namentlich die kriminelle Persönlichkeit, bezogen. In der Tendenz mutiert Sicherheit als agency heutzutage zu einer Größe, von der öffentlich gesprochen wird, als könne sie die ganze scene prägen, denn ihr Skopus erweitert sich stetig. Bei Burke findet sich für diese semantischen Räume, die in sich alle Bereiche öffentlichen Sprechens einschließen, der Ausdruck "szenischer Begriff" (Burke 1969b: 134-135). Die zyklisch wiederkehrenden, szenischen Problematisierungen der Sicherheit rufen stets die Ereignisse in der internationalen Politik hervor, aber auch kleinere auf Sicherheit beziehbare "Zwischenfälle".

Genauer wird hier die Verwendung des Begriffs "Krise" erläutert. Zunächst lässt sich feststellen, dass die Verwendung des Begriffs Krise durch die Polizei an mangelnde rechtliche Vorgaben gebunden ist. Wann genau eine Krise eintritt, definiert das BBK folgendermaßen:

Wenn Gefahren- und Schadenslagen sich derart zuspitzen, dass die alltäglichen Maßnahmen und Mittel für die Vermeidung und Reduzierung von Schäden nicht ausreichen, handelt es sich um eine Krise. Die notwendigen Anpassungen zur Vermeidung von, Vorbereitung auf, Erkennung und Bewältigung sowie Nachbereitung von Krisen werden als Krisenmanagement bezeichnet.

(Bundesamt für Bevölkerungsschutz und Katastrophenhilfe 2005)

Das Krisenmanagement erlaubt weitreichendere Eingriffe als andere Gefahrenlagen. Kavala sprach vom G8 weniger explizit als Krise, jedoch bediente sie in ihrer Öffentlichkeitsarbeit stets die Konstituierung einer Krise, indem sie das Vorhandensein derselben antizipierte. Bereits 2006 stand in einer internen Mitteilung: "Bereits mehrfach wurde im Presseausschuss des Deutschen Städtetages die Möglichkeit der gegenseitigen Amtshilfe von Pressestellen in Krisensituationen thematisiert." (13 IM OBR VS, 24.04.2006) Und auch in diesem Kontext wird die Rolle der Öffentlichkeitsarbeit keineswegs unterschätzt: "Die Glaubwürdigkeit des Krisenmanagements hängt entscheidend davon ab, wie effektiv die Öffentlichkeitsarbeit funktioniert." (13 IM OBR VS, 24.04.2006) Die Zusammenarbeit der Pressestellen zwecks einer einheitlichen Darstellung dieser Krise wurde kurz darauf angefordert - das Eintreten des Krisenfalls für Heiligendamm vorausgesetzt. In der Öffentlichkeit sprach erst im Januar 2007 der Innenminister M.-V. Lorenz Caffier direkt von einer Krise: "Die Beauftragten der Bundeswehr für zivil-militärische Zusammenarbeit arbeiten bereits aktiv in den eingerichteten Krisenstäben der betroffenen Landkreise mit. Es hat sich gezeigt, dass diese Strukturen Akzeptanz finden. Diese Zusammenarbeit ist zukunftsträchtig" (206 PM MdI M.-V., 17.01.2007). Die zivil-militärische Zusammenarbeit erforderte zu diesem Zeitpunkt noch eine Legitimation über eine Krise - ein Rechtsgrundsatz, der sich zunehmend auflöst.

Denormalisierungsängste als Ängste vor einer Krise wurden über Pressemitteilungen im mediopolitischen Diskurs geschürt und sogleich beruhigt. Denn "[e]s gehört zu jeder Krise, dass sie als außerordentlich und dramatisch erlebt wird" (Rühle/Brendecke 2011). Kavala sah dies als Teil ihrer Aufgabe, den G8-Gipfel nicht nur mit einem "Krisenmanagement", sondern zudem mit einer strategischen "Krisenkommunikation" zu begleiten, um "Medien-Hypes" zu verhindern (13 IM OBR VS 24.04.2006). 
Legitimierung von sonder- und außerrechtlichen Maßnahmen

Für die Implementierung rechtlicher Neuerungen war mediale Präsenz notwendig. Diese konnte Kavala erfolgreich durchsetzten, wie v. a. die Analyse des medialen Diskurses von Selz (2008) zeigt. Wie er nachweisen konnte haben sich die Presseagenturen vor allem auf die Aussagen Kavalas verlassen. Die Meldungen der Agenturen sind nicht nur ausschlaggebend für die mediale Vermittlung des Ereignisses in die Öffentlichkeit, sondern haben ebenfalls Einfluss auf die hegemoniale Wahrnehmung.

Abbildung 5: Prozentuale Verteilung der Bewertungen der Demonstranten

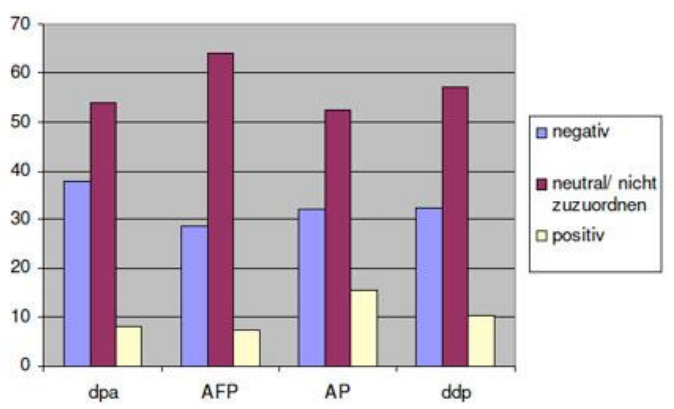

Abbildung 24: Verhältnis negativer und positiver Aussagen der Korrespondenten über die Demonstranten

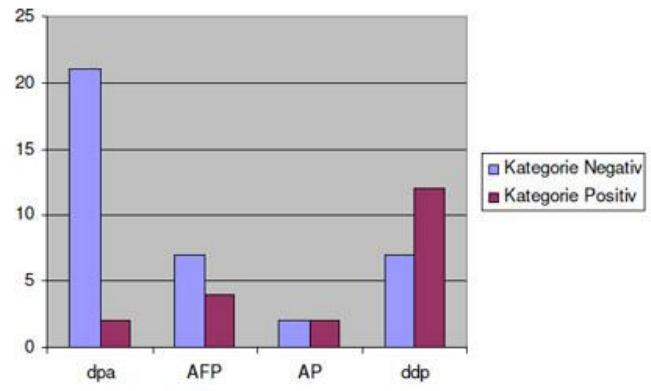

Abbildung 3: Präsenz in Agenturmeldungen. Quelle: Selz 2008

Dazu wurden nicht nur die expliziten LAGEbegriffe benutzt, sondern stereotype Krisenmetaphern verwendet: "Frühwarnungen", "Weichen stellen", "Spirale der Gewalt". Schließlich bestätigt sich, was auch zahlreiche AutorInnen vermuten: Dass Krise nicht einfach ist, sondern gemacht werden muss. "Krise ist keine Sache, sondern eine Beschreibungsoption. Sie wird diagnostiziert, ausgerufen, hergestellt" (Rühle/Brendecke 2011). Diese Bilder sind exemplarisch für normalistische Interdiskurse, in denen Krisen als soziale Gegenstände konstruiert werden (vgl. Parr 2009). Neben der Dramatisierung der LAGE wurde stetig betont, dass die Behörden der Bedrohung gewachsen seien. Caffier schrieb bereits vor dem Gipfel in einem Grußwort an die Polizeikräfte: "Ich bin sicher, dass Sie gemeinsam mit den Kräften unserer Landespolizei die anstehenden Aufgaben bewältigen". (BAO Kavala 2007: 2) Diese Entdramatisierung wurde sowohl nach innen als auch nach außen an die Öffentlichkeit gerichtet kommuniziert.

Aus diskursanalytischer Perspektive ist der Krisenbegriff in mehreren Schritten deutlich fassbar. Diese Schritte fasst Habscheid folgendermaßen zusammen:

Als Verlaufsbegriff ist die "Krise" narrativ verfasst: Sie markiert eine gute Vergangenheit, zeichnet einen Verfall nach, flaggt die Gegenwart als liminale Übergangszeit aus und entwirft hierin tritt ihr spezifisch moderner Zuschnitt in den Vordergrund - optionale Zukünfte. Dabei ist der Krisenbegriff, wie auch der Katastrophenbegriff, auf mediale Repräsentationen angewiesen.

(Habscheid/Koch 2014)

Aus dieser Darstellung, die auch für den Diskurs um Heiligendamm nachweisbar ist, leitet Habscheid ab, wie diese Narration funktionalisiert wird:

Der Krisenbegriff ist diagnostisch angelegt, er impliziert eine Appellstruktur ("Tut doch was!") und bündelt divergierende Elemente einer historischen Konstellation zu einem Verlauf. Dabei transportiert er kulturelle und politische Konnotationen und beeinflusst - oftmals im Rücken der Diskursteilnehmer - die Wahrnehmung und Bewertung von Ereignissen im Sinne einer retroaktiven Performativität (vgl. Žižek 1992). Nicht zuletzt kann sich der Krisenbegriff auch gegen seine eigenen Redebedingungen wenden. Dort, wo er seine eigene Möglichkeit - als Rede von 
der Krise der Zeichen, der Krise der Wahrnehmung, der Krise des Denkens - bestreitet, gewinnt er an Eindringlichkeit, wird der Reaktions- und/oder Anpassungsdruck erhöht.

(Habscheid/Koch 2014)

Sowohl für rechtliche Texte als auch für die Kommunikation Kavalas lässt sich eine ähnliche Struktur zeigen: Die Krise ist stets als Verlauf angelegt und führt zur Erzwingung von $\mathrm{Zu}-$ stimmung für besondere Maßnahmen. Der Topos der Krise - ursprünglich das entscheidende Stadium einer Erkrankung - impliziert eine "Körperlichkeit" des Ereignisses, einen Körper, dem etwas diagnostiziert wird. Der Begriff Krise steht dabei der "Gesundheit" gegenüber (vgl. Grunwald/Pfister 2007). Diese Gesundheit ist in der verdateten Gesellschaft gleichgesetzt mit Normalität. Die Krise wird als ein rhetorisches Werkzeug der Inszenierung konkreter politischer Prozesse im Raum des öffentlichen Diskurses eingesetzt. Krise diagnostiziert stets einen Notstand, implementiert dringenden Handlungsbedarf aufgrund von Zeitmangel. Wer von Krise spricht und Krise manifestiert, inszeniert sich selbst als erkennend. Wer als ersteR von der Krise spricht und sie damit manifestiert, kann später behaupten, sie als ersteR erkannt zu haben. Wer eine Krise beschwört, konstruiert damit zugleich eine Bühne für Krisenhelfer wenig verwunderlich, dass Beschwörer und Retter erfahrungsgemäß dieselben AkteurInnen sind.

\subsection{Purpose: friedliche Demonstranten}

Friedliche Demonstranten sind im Sprachgebrauch Kavalas keine agents, sondern werden als purpose vorgegeben. Die unterschiedlichen Mechanismen der FEINDBILDkonstruktionen, die zur Legitimierung der Einsätze handhabbar gemacht wurden, werden in der Analyse stets diesem purpose der friedlichen Demonstranten gegenübergestellt. Dieser Topos umfasst diejenigen Personen, die im Rahmen der verfassungsrechtlichen Möglichkeiten ihre Meinung oder auch ihren politischen Widerspruch kundtun, allerdings mittels Formen des Protestes, die hegemonial als legitim anerkannt sind - sprich: gewaltlos handeln. Mit diesem Topos diskursiv eng verknüpft werden die Grundrechte, die friedliche Demonstranten in Anspruch nehmen. Sie stünden auf der Seite von Recht und Gesetz, heißt es, seien "friedlich und legitim" (MdI M.-V., 13.06.07), handeln im legalen Rahmen, solange sie die Anweisungen der Polizei befolgen. Sie dienen in den Begründungslogiken der Einsätze als Medium für Hochwerte wie Sicherheit und Rechtsstaatlichkeit. Kavala schreibt: "Wir erwarten viele friedliche Meinungsäußerungen und werden diese Demonstrationen vor unfriedlichen und gewaltbereiten Kundgebungsteilnehmern schützen" (Kavala Report 2007: 4). Die Gleichsetzung von friedlich und legitim zeichnet alle Kommunikationen der Behörden aus. Friedliche Demonstranten kommen in der internen Kommunikation der unterschiedlichen Polizeieinheiten an keiner Stelle vor. In der Kommunikation Kavalas mit anderen Behörden und in der öffentlichen Kommunikation werden friedliche Demonstranten ausschließlich als Opfer von gewalttätigen Demonstrierenden angeführt, nie als aktiv handelnde Personen.

Die Polizei inszeniert sich als neutralen Schiedsrichter. In diesem Zusammenhang werden die friedlichen Demonstranten dazu aufgefordert, sich "von Extremisten zu distanzieren" (27 MA Kavala EA3, 12.06.2007). Jedoch verändert das BKA nach der Demonstration am 02.06.2007 die öffentliche Darstellung der Demonstrationen: 
Legitimierung von sonder- und außerrechtlichen Maßnahmen

Nach der Fokussierung auf den 'Schwarzen Block' im Zusammenhang mit den Ereignissen vom 02.06.2007 kleideten sich Teile der gewaltbereiten Störer nicht mehr schwarz, was eine Differenzierung zu den friedlichen Demonstranten deutlich erschwerte.

(BKA 2007: 29)

Mit diesem Moment nimmt die polizeiliche Presse- und Öffentlichkeitsarbeit von dem Topos Abstand und nimmt stattdessen eine differenziertere Beschreibung der potenziellen Gefahrenverursacher vor.

\subsection{Agents: Feinde}

Die FEINDE sind in der Darstellung Kavalas die für Gefahr und Risiko verantwortlichen agents. Diese Darstellung erlaubt Kavala, eigene Verantwortlichkeiten als notwendige Reaktionen $\mathrm{zu}$ bezeichnen. Die agents bestimmen also die agency der contra-agents. FEINDBILDER dienen im Korpus der Kontrastierung von Hochwerten, die Beschreibungen dieser FEINDE in den Aussagen Kavalas sind sehr unterschiedlich - auch wenn sie in ihrer Konsequenz allesamt eine Konstitution von Gefahr beinhalten. ${ }^{11}$

Eine zentrale Rolle in der internen Kommunikation von FEINDBILDERN spielt das Ampelsystem. Da die Weitergabe von Informationen in bedrohlichen Situationen während eines Polizeieinsatzes schnell gehen muss, wird z. B. über Funk und in kurzen Mitteilungen eine Zuweisung der Demonstranten in die drei Kategorien des Ampelsystems verlangt. In die Kategorie "rot" fallen als "gewaltbereit" eingestufte Personen, deren Ziel die Ausübung von Gewalt und das Begehen von Straftaten ist. Diese Gruppe wird dadurch entpolitisiert, dass ihnen Gewaltausübung als primäre Motivation zur Teilnahme an Demonstrationen unterstellt wird. Zur Klassifizierung "rot" reicht unter anderem die Distanz der Demonstration zum Wohnort. Es gibt offenbar keinen Grund, dieser Gruppe die Teilnahme zu ermöglichen oder zu erlauben (vgl. Ullmann 2007: 33). Mit der Kategorie "gelb" werden die Personen charakterisiert, die nicht eigenständig Gewalt ausüben, allerdings leicht verführbar seien - MitläuferInnen, SympathisantInnen. Auch hier wird diskursiv eine Reduktion auf das Gewaltpotenzial vorgenommen. Mit der Einstufung "grün" werden alle übrigen Personen erfasst. Dieser Gruppe wird zwar keine direkte Gewaltbereitschaft zugeordnet, jedoch die Bereitschaft nicht ausgeschlossen, anderen Personen Übergriffe zu ermöglichen. Indem sie diese decken, so heißt es in internen Unterlagen, bieten sie Gewalttätern gegebenenfalls Schutz. In den Funksprüchen und Meldungen wurde stets jeder Person eine Kategorie zugewiesen. So wurden alle TeilnehmerInnen zu einer potentiellen Gefahr. Die Abgrenzung zwischen friedlichen und gewalttätigen Demonstranten wird damit unterlaufen, weil alle Beteiligten an den Demonstrationen eine potentielle Gefahr ausstrahlen. Schon im Vorfeld des Gipfels, also ohne den akuten zeitlichen Druck, wird in der internen Kommunikation diese Klassifikation vorgenommen.

\footnotetext{
${ }^{11}$ Auch wenn der Feindbegriff damit eine zentrale Position einnimmt, verstehe ich mich keinesfalls als Schmittianerin. In Abgrenzung von Schmitt wird der Terminus FEIND hier als frame-Benennung im Burke'schen Sinne verwendet.
} 


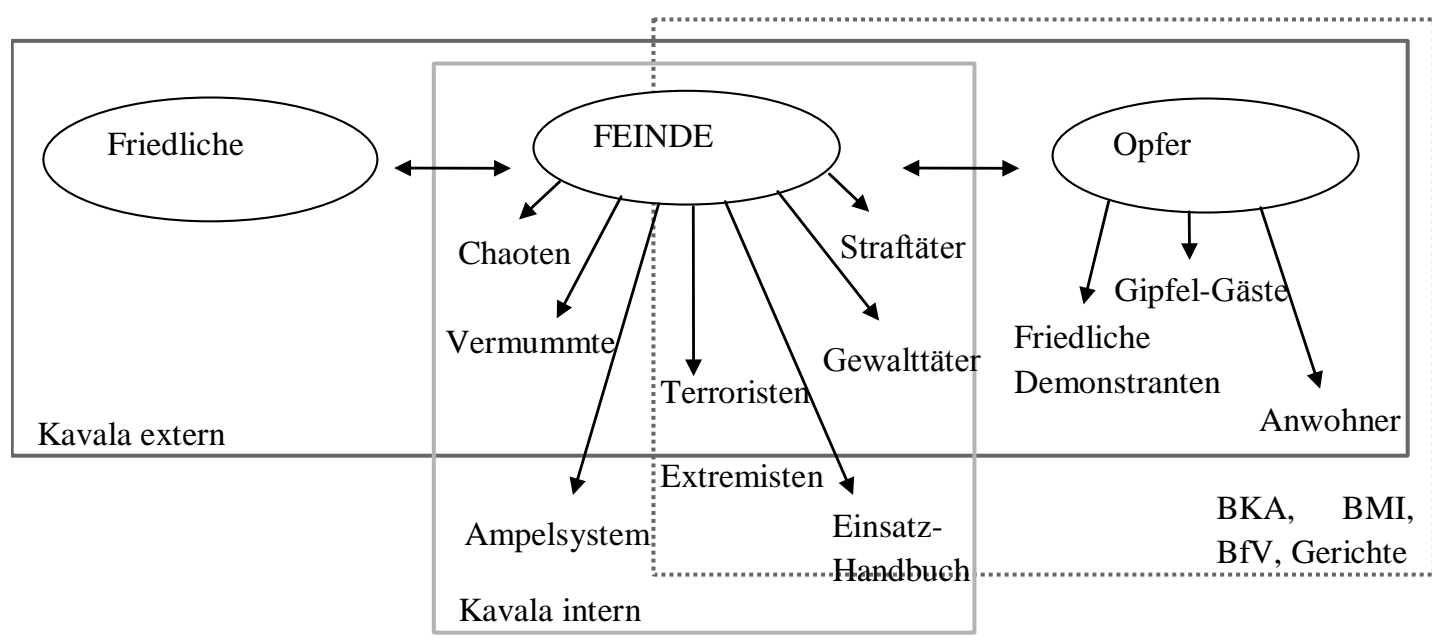

Abbildung 4: Toposstruktur feindliche und friedliche Demonstranten. Quelle: Eigene Darstellung

Der Topos der Straftäter ist aus zwei Aspekten besonders interessant. Zum einen, weil eine Straftat die Grundlage für richterliche Entscheidungen ist, zum anderen weil Kavala dem Begriff eine Bedeutung anhängt, die dieser Bezeichnung weder im juristischen Kontext noch im Alltagsverständnis zukommt. Als Straftäter bezeichnet Kavala in ihrem Einsatz in Heiligendamm - entgegen der juristischen Bedeutung, die dem Alltagsverständnis des Begriffs nahe kommt - keine Person, die Straftaten begangen hat, sondern eine Person, die verdächtigt wird, Straftaten zu begehen. In der Pressemitteilung des IM M.-V. vom 13.06.07 heißt es: "Nach ersten Feststellungen wurden am 2. Juni in Rostock durch die Polizei 95 Straftäter festgenommen" (Innenministerium Mecklenburg-Vorpommern, 13.06.07). Immerhin zehn Tage nach den erwähnten Ereignissen vom 02.06.07 klingt diese Mitteilung zunächst schockierend. Die Zahl wirkt klar als Aussage, dass fast 100 Täter gestellt werden konnten. Diese Zahl lässt auf eine enorme Anzahl von Taten und TäterInnen schließen. Zudem kann davon ausgegangen werden, dass nur ein Teil der TäterInnen tatsächlich gefasst werden konnte. Diese Aussage wird durch die zeitliche Distanz zum Ereignis als glaubwürdiger, verifizierter Tatsachenbericht zitiert. Allerdings gab es im Anschluss lediglich 9 Haftbefehle und 24 Gewahrsamnahmen. Nicht allen 95 festgenommenen Straftätern konnten demnach Straftaten nachgewiesen werden. Durch diesen unpräzisen Gebrauch wird eine Vorverurteilung gegen die Personen vorgenommen. Die Öffentlichkeitsarbeit Kavalas folgt Empfehlungen zur Steigerung der Legitimität, wie sie in einem Handbuch, das europaweit zu Schulungen vor großen Demonstrationen eigesetzt wird, gegeben werden. "Streben Sie eine größere Zahl von Strafverfahren an", rät dieses Arbeitspapier von EU-SEC den Polizeien der EU bei Großereignissen (Council of the European Union 2004). ${ }^{12}$ Bereits in einem Bericht im Vorfeld der Gipfelproteste warnt Kavala vor diesen zu erwartenden Straftaten. Die Straftaten, auf die dabei referiert

\footnotetext{
${ }^{12}$ Das Programm "Coordinating National Research Programmes on Security during Major Events in Europe" (EU-SEC) wurde zunächst von 2004 bis 2008 von allen europäischen Ländern auf Anraten der EU-Kommission für Forschung und Innovation im Rahmen des ERA-NET Programms durchgeführt und dann verlängert. Es vergleicht die Einsatzmöglichkeiten der Polizeien bei Großevents und unterstützt die einzelnen Länder. Im Rahmen der Zusammenarbeit wurde 2005 die Europäische Polizeiakademie (EPA) gegründet. Sie setzt die Erfahrungen um und verordnet sich der Stärkung der Zusammenarbeit der nationalen Behörden auf dem Gebiet der Verbrechensbekämpfung und -vorbeugung.
} 
Legitimierung von sonder- und außerrechtlichen Maßnahmen

wurde, waren "Brandanschläge auf Fahrzeuge und Sachbeschädigungen" im Vorfeld des Gipfels. Kavala schrieb: "Diese unfriedlichen Aktionen zeigen, dass militante G8-Gegner auch in Bezug auf den Gipfel in Heiligendamm vor der Ausübung schwerster Straftaten nicht zurückschrecken" (BAO Kavala 2007: 15). Die Klassifizierung der Straftaten als "schwerste" im Zusammenhang mit Sachbeschädigung stößt sich an der juristischen Verwendung des Begriffs Straftat. Zum einen wird in keinem Gesetz dieser Superlativ verwendet, sondern lediglich das Adjektiv schwer. Zum anderen wird die Bewertung einer Straftat als "schwere" Straftat laut StGB vor allem im Kontext von Hausfriedensbruch (StGB § 123), Landfriedensbruch (StGB § 125), Sexual- oder Gewaltdelikten wie auch Raub oder gemeingefährlichen Taten (StGB § 167a, § 213, § 226) verwendet - weniger in Zusammenhang mit Sachbeschädigung.

Die Prognose Kavalas diente darüber hinaus dazu, Panik vor dem Protest zu verbreiten. Die Spaltung in friedliche und gewaltbereite Demonstranten wurde auch vom MdI M.-V. vertreten und die Definition von Straftaten in den Bewertungen der Handlungen induziert: Der Wurf von Farbe auf ein Hotel liefert eine erste Rechtfertigung der langen Vorbereitung auf den Einsatz, weil er als Straftat die Aktion in den Kontext der Gewaltausübung rücken ließ. Caffier: "Ich habe Verständnis für einen friedlichen Protest von Gegnern des G8-Gipfels. Kein Verständnis habe ich für Aktionen dieser Art [...]." (205 PM MdI M.-V., 28.12.2006) Deutlicher äußert er sich kurz vor dem Gipfel: "Friedlicher Protest ja, Gewalt nein!" (207 PM MdI M.-V., 22.05.2007) In Bezug auf den Topos der Straftäter ist eine direkte Verbindung zwischen Kavala und den verantwortlichen Gerichten zu erkennen. So haben z. B. Falschmeldungen Kavalas über die angebliche Bewaffnung der TeilnehmerInnen einer nicht genehmigten Demonstration an der Galopprennbahn eine Straftat konstruiert. Die angebliche Bewaffnung ist bereits ein ausreichender Straftatbestand, der das Einschreiten von Polizei sowie von Gerichtsverfahren erlaubt. In den Mitteilungen, Urteilen und Urteilsbegründungen kommt vor allem der sachlich wirkende Begriff Straftäter vor (vgl. OLG Rostock, 28.08.2007). Die problematische Verwendung des Begriffs durch die Polizei zeigt sich schließlich auch daran, dass das BKA nach Recherchen der Zeit unter der Bezeichnung "Straftäter" bis heute eine Kartei führt, in der tausende Menschen, die weder straffällig gewesen seien, noch Vorstrafen oder offene Verfahren anhänglich hätten, registriert werden (vgl. Zeit online 2014). Diese Kartei lässt unzählige Delikte vermuten. Es wird jedoch keine Straftat vorausgesetzt. Das BKA erklärte auf Nachfragen mehrerer JournalistInnen nach dem Begriff Straftäter, dass der Begriff intern schlicht dazu benutzt werde, eine klare Abgrenzung gegenüber der Opfergruppe herzustellen.

Diese Konstruktion von FEINDBILDERN ermöglichte die Legitimation von Notstandsmaßnahmen, indem zuerst ein öffentlich wirksames FEINDBILDrepertoire geschaffen wurde, dessen Ablehnung zustimmungspflichtig war. Dieses wurde dann im zweiten Schritt durch gezielte Öffentlichkeitsarbeit und selektiv dramatisierende Darstellungen der Demonstration am 2. Juni bestätigt. Anhand dieser Wirklichkeitskonstruktionen konnten Verbote und Einschränkungen für den Verlauf der Gipfelproteste erwirkt und langfristig manifestiert werden.

\subsection{Sprache und Recht}

Umfassende rechtliche Neuerungen wurden durch diesen diskursiv erzeugten Bedrohungszustand in Heiligendamm durchgesetzt, diskursiv wurde über die mediale Krise der rechtliche 
Ausnahmezustand implementiert. Die zentralen Ergebnisse der Untersuchung von Ausnahmerhetorik als Prävention und Reaktion sind in der folgenden Tabelle zusammen gestellt. Die Sprechmuster des Ausnahmezustands in ihrer jeweiligen Funktion als Prävention (angesichts eines Risikos) werden mit denen als Reaktion (angesichts einer Gefahr) verglichen. Im Kontext des zukünftigen Risikos wurde eher von einer allgemeinen Bedrohung, vor allem durch Terrorismus gesprochen. Kavala informierte wissentlich falsch über zu erwartende Taten. Dabei wurde der Sagbarkeitsrahmen vor allem durch die Rede vom Schutz des Gipfels als Ziel bestimmt und die Legislative damit beauftragt, Gesetze dahin gehend zu ändern, der Exekutive möglichst breite Handlungsrahmen zu schaffen. Im Kontext der gegenwärtigen Gefahr hingegen wurden konkrete Straftäter konstruiert, darunter Clowns und Vermummte.

\begin{tabular}{|r|l|}
\hline$\underline{\text { Risiko }}$ & \multicolumn{1}{c|}{ Gefahr } \\
Allgemeine Bedrohung & Konkrete Straftäter \\
Terrorismus & Clowns, Vermummte, Störer \\
eher scene dominiert & eher agent dominiert \\
Zukünftig & Gegenwärtig \\
Falschmeldungen über Verdachtsmomente & Falschmeldungen über Straftaten \\
Fokus auf Schutz des Gipfels & Fokus auf Angriff gegen die Polizei im Einsatz \\
Legislative & Judikative \\
\hline
\end{tabular}

Abbildung 5: Kavalas Sprachmuster des Ausnahmezustands. Quelle: Eigene Darstellung

Aus diesen Beobachtungen lässt sich die These ableiten, dass die Konstitution des Ausnahmezustands in seiner Funktion der Gefahrenreaktion vom präventiven Eingriff erweitert oder auf lange Sicht sogar abgelöst wird. Semantisch wird der Ausnahmezustand umkodiert zu einem konstruierten Ereignis, das als Reaktion "künstlich" erschaffen wird, während dessen allerdings das Gouvernement die Kontrolle behält.

Caffier drückte im Vorfeld deutlich die Richtung aus, in die der Gipfel gelenkt werden sollte: "Zur Abwehr dieser Gefahren [durch Extremismus; A. d. V.] sind Bund und Länder ständig bemüht, die Gesetzlage den zunehmenden Gefahren anzupassen, um den Sicherheitsbehörden rechtzeitige und angemessene Gegenmaßnahmen zu ermöglichen." (207 PM MdI M.-V., 22.05.2007) Das Gesetz musste der Effizienz der Exekutive weichen. Der Hochwert "Sicherheit" legitimierte nicht nur weitreichende Änderungen des Gesetzes, sondern diente vielmehr als Leitwert, an den das Gesetz angepasst werden sollte. Kavala verpflichtete z. B. RichterInnen des Amtsgerichts Rostock zu "Übungen", über deren Inhalt sie "Schweigepflicht" einzuhalten hatten (vgl. Backmund et al. 2007). RichterInnen des Landgerichts M.-V. trugen während des Gipfels Anstecker mit der Aufschrift "Kavala Justiz" (vgl. Backmund et al. 2007) eine Symbolik, die jegliche Annahmen von Gewaltenteilung verhöhnt.

Um weitreichende Maßnahmen gegen die potentiellen Störer ergreifen zu können, erwirkten die Behörden unter Leitung Caffiers und Kavalas bereits im Vorfeld die Neuerung des SOG M.-V. auf Landesebene. Durch die Legitimierung verschiedener Maßnahmen in diesem Gesetzestext wurde Heiligendamm zum Experimentierfeld. Die Horrorszenarien von gewalttätigen Autonomen erklärten die vermeintliche Notwendigkeit der präventiven Videoüberwa- 
Legitimierung von sonder- und außerrechtlichen Maßnahmen

chung, Telefonüberwachung, DNA-Analyse und der verdachtsunabhängigen Erfassung von PKW-Kennzeichen u. v. m. Der besondere Fall, in dem das SOG M.-V. diese Maßnahmen genehmigt, bedarf der (Konstruktion einer) konkreten Gefahr als Legitimation. Diese Neuerung des SOG M.-V. war das Projekt der regierenden Koalition aus SPD und Linkspartei unter Innenminister Gottfried Timm (SPD). Damals hieß es, die Maßnahmen wären zeitlich befristet und nur zur Durchführung des Gipfels angekündigt. 2011 wurde von der neuen konservativen Landesregierung aus CDU und SPD mit einem Änderungsgesetz zum SOG M.-V. der Ausnahmefall entfristet. Die ehemals zeitlich beschränkten Maßnahmen aus dem Polizeigesetz wurden entfristet und so der Ausnahmefall anlässlich des G8-Gipfels zum permanenten Ausnahmezustand. M.-V. hat seitdem eines der härtesten Polizeigesetze in der BRD (vgl. Landtag Mecklenburg-Vorpommern 2011: 1). Auch das LMG M.-V. wurde bereits im Juli 2006 geändert, um den Zugang zu weiterführenden personenbezogenen Daten - darunter frühere Namen, Staatsangehörigkeit, Familienstand und Anschriften - zu erleichtern (2 IM Kavala SHB, 16.01.2007).

Das BMI hatte nicht nur bereits im Vorfeld angekündigt, Grenzkontrollen zuzulassen, sondern auch Einreiseverbote entgegen der Schengener Abkommen zu veranlassen:

Aufgrund der erhöhten Sicherheitsanforderungen anlässlich des G8-Gipfels in Heiligendamm/Mecklenburg-Vorpommern vom 6. bis zum 8. Juni 2007 hat Herr Bundesinnenminister Dr. Schäuble entschieden, vorübergehend Grenzkontrollen an den Binnengrenzen wieder aufzunehmen $[\ldots]$.

(28 IM BMI VS, 27.04.2007)

$\mathrm{Ob}$ in der temporären Aufhebung der Schengener Abkommen bereits ein Verstoß gegen europäisches Recht besteht, bleibt umstritten. ${ }^{13}$ In jedem Fall entsteht so ein temporärer Ausnah-

\footnotetext{
${ }^{13}$ Konkret lässt sich dies am Beispiel der Internationalisierung der Sicherheitspolitik im europäischen Raum zeigen. 1998 griffen deutsche Hooligans während der Fußballweltmeisterschaft einen französischen Polizisten an. Er ist seitdem querschnittsgelähmt. In Folge wurden verstärkte Kommunikation der europäischen Sicherheitsbehörden und eine gemeinsame Strategie verabredet. Die EU setzt seitdem regelmäßig einige Tage vor Beginn von relevanten Veranstaltungen Kontrollen der Schengen-Grenzen ein. Womöglich reisewillige Fußballfans, aber auch DemonstrantInnen, wurden von Behörden des jeweils eigenen Landes an der Ausreise gehindert. Bereits anlässlich der Proteste 2001 in Genua schrieb die GdP in einer PM:

"Wer nur die Absicht hat, im Ausland mit brachialer Gewalt und radikaler Brutalität politische Gespräche zu verhindern, muss zu Hause bleiben. [...] Schon bei der letzten Fußball-Europameisterschaft in den Niederlanden und Belgien hatten sich verschärfte Grenzkontrollen, pass- und melderechtliche Beschränkungen und von der Polizei geführte so genannte Gefährderansprachen für gewaltbereite Fußballanhänger als sehr erfolgreich erwiesen. Freiberg: 'Diese Hooligans haben ihr Ziel - in jeder Hinsicht - nicht erreicht. Dies muss uns auch bei den Polit-Hooligans gelingen."' (GdP 20.06.2007).

So konnten die Reiseverbote nicht nur an deutschen Grenzen legitimiert werden, sondern auch an anderen europäischen Grenzen. Die Reiseverbote waren zumindest vor dem G8 in Heiligendamm in der deutschen Rechtsprechung noch umstritten:

"Wegen der sehr hohen Sicherheitsanforderungen vertrat das BMI bereits im Begleitkonzept [...] die Auffassung, ausländerrechtliche Maßnahmen seien in der Regel nicht geeignet, Unionsbürger, die als Gefährder bekannt sind, aus rein generalpräventiven Gründen an der Einreise und dem Aufenthalt im Bundesgebiet zu hindern. Sicherheitsprobleme (während der WM 2006 - und jetzt -ggf. zum G8-Gipfel) seien daher ausländerrechtlich nur schwer zu lösen; es müsse vielmehr auf die Instrumentarien des Polizei- und Ordnungsrechts zurückgegriffen werden." (20 RB MdI M.- V., 11.01.2007). Diese Bedenken konnten durch die Praxis in Heiligendamm ausgeräumt werden.
} 
mezustand, in dem gegen Grundrechte verstoßen wird, um besondere Maßnahmen zu ermöglichen. Interessant ist dieses Beispiel, weil es einen gängigen - normalisierten - Verstoß gegen internationale Abkommen darstellt: Nicht zuletzt anlässlich der Fußball-WM in Deutschland im Jahr 2006, aufgrund der sogenannten "Flüchtlingskatastrophe" und wegen politischer Großveranstaltungen wurde dies zur Regel. Dieser Ausnahmeregel wurde 2013 mit der "Notfallregel für den Schengen-Raum" zudem eine neue Rechtsgrundlage gegeben. Nicht zuletzt durch diese Normalisierung der Ausnahme stehen die europäischen Verträge zur Debatte und werden "justiert" - was nichts anderes bedeutet, als dass ihnen permanent Möglichkeiten von Ausnahmemaßnahmen eingeschrieben werden. Was in Heiligendamm getestet wurde, konnte schließlich vom EU-Parlament in passende Gesetze gegossen werden. Mit dem Hinweis auf eine Gefahr der Sicherheit und Ordnung kann die im EU-Recht verankerte Bewegungsfreiheit ausgesetzt werden. Über das Argument des "letzten Mittels" wurde der Öffentlichkeit suggeriert, es handele sich weiterhin nur um Maßnahmen des Notfalls:

Der gemeinsame Vorstoß von Innenminister Claude Guéant und seinem Kollegen Hans-Peter Friedrich klingt doch harmlos, zumindest nach Augenmaß und Vernunft. Der Vorschlag: Die EU-Regierungen sollen ihre Grenzen künftig wieder kontrollieren, sprich: abriegeln dürfen. Natürlich nur als letztes Mittel, versteht sich.

(Boehme 2012)

Das Europäische Parlament zögerte nicht lange, dem Vorschlag zuzustimmen und die neuen Regeln für den Schengen-Raum zu legalisieren. Im Sinne des Linkschen "Normalismus"Konzepts entsteht Normalität diesseits expliziter Normierungen, indem das Parlament "legalisiert" was bereits "normalisiert" war. Die Möglichkeiten für Kontrollen an innereuropäischen Grenzen wurden dadurch deutlich ausgeweitet: EU-Staaten dürfen künftig Kontrollen nicht nur - wie bereits faktisch der Fall war - bei Terrorangriffen oder Großveranstaltungen, sondern auch bei "schwerwiegenden Defiziten bei der Kontrolle der Außengrenzen" und einer Gefahr für Sicherheit und Ordnung durchführen (BMI 08.10.2013). Die Mitgliedsstaaten definieren diese Gefahr im Übrigen selbst. Nach dem bisher Gesagten zum Topos Gefahr und den Hochwerten Sicherheit und Ordnung muss diese Neuregelung als die schleichende Implementierung des Ausnahmezustands interpretiert werden. ${ }^{14}$

\section{$4 \quad$ Ergebnisse}

Die Kategorisierungen der Polizei, die zunächst der kommunikativen Handlungslogik von Einsätzen geschuldet zu sein scheinen, führten anlässlich des Gipfeltreffens 2007 dazu, dass die ehemals zeitlich beschränkt vorgesehenen Maßnahmen des Polizeigesetzes entfristet wurden und so der Ausnahmefall anlässlich des G8-Gipfels zum permanenten Ausnahmezustand im Sinne Agambens werden konnte. Die Kommunikation staatlicher Akteure erhob sich dazu aus den ihr anverwandelten Zuständigkeiten, so wie Kavala an der Formulierung des SOG

\footnotetext{
${ }^{14}$ Kritik kam u. a. von der EU-Fraktion der Grünen: "Erstmal Grenzen zumachen, die Begründung finden die Staaten dann schon. Diese Möglichkeit macht mir Angst", sagte Ski Keller: "Letztlich werden diese Regeln zur Ausweitung von Grenzkontrollen führen." (Die Grünen, Pressemitteilung, www.gruene-europa.de/neue-regelnfuer-den-schengenraum-10032.html) Ihre Befürchtung liegt nahe. Schließlich ist die Ausweitung der Grenzkontrollen hiermit gesetzlich verankert worden und es wäre schließlich nicht der erste Fall, in dem als Notfall-Mechanismus bezeichnete Maßnahmen zur Regel werden.
} 
Legitimierung von sonder- und außerrechtlichen Maßnahmen

M.-V. beteiligt war und auch die Rechtsprechung beeinflusste. Kavala hatte demnach Einfluss auf die Definition dessen, was hegemonial als legitim erachtet wird. Die strategische Kommunikation kann besonders deutlich an der Konstituierung von Ausnahmezuständen seitens der Exekutive zur Rechtfertigung ihres Einsatzes gezeigt werden. Am Beispiel des Protestes zum G8-Gipfel in Heiligendamm lassen sich exemplarisch zum einen die vorbereitende Konstruktion von präventiven Legitimierungsoptionen ${ }^{15}$ und zum anderen die anschließend tatsächlich realisierten Rechtfertigungsstrategien aufzeigen.

Die Rolle der Medien in diesem Beispiel für Grauzonen der Demokratie lässt sich besonders anhand der Relevanz der Presseagenturen nachzeichnen. Selz (2008) konnte u. a. nachweisen, dass die Behörden und vor allem die Polizei übermäßige Präsenz gegenüber anderer Stimmen in den Medien hatten. Daraus lässt sich schließen, dass die Polizeipressestelle eine kaum hinterfragte diskursive Hoheit genoss. Die Pressemitteilungen der Polizei wurden von Agenturen wie einzelnen Redaktionen als verlässlich, wenn nicht gar als objektiv eingeschätzt und unhinterfragt verbreitet. Falschmeldungen seitens der Polizei wurden übernommen. Und selbst wenn polizeiliche Falschmeldungen von der Presse aufgedeckt worden sind, führte dies zu keiner offiziellen Korrektur seitens der Polizei (vgl. Rucht/Teune 2008). Kavala - allen anderen Behörden voran - beförderte öffentlich ein Klima der Angst zu implementieren, welches dann sonderrechtliche Maßnahmen als legitim erschienen ließ. Ihre diskursive Strategie zielte auf die strategische Manifestierung von Denormalisierungsängsten ab.

\section{Literatur}

Agamben, Giorgio (2004): Ausnahmezustand. Homo sacer II.1. Frankfurt a. M.: Suhrkamp. Agamben, Giorgio (2014): "Vom Kontrollstaat zur destituierender Macht. Wie im permanenten Ausnahmezustand regiert wird". Aus dem Englischen von Thomas Laugstein. Luxemburg 1/2014: 28-33.

Backmund, Michael/Donat, Ulrike/Ullmann, Karen (2007): "Feindbild Demonstrant. Polizeiliche Desinformationspolitik in Heiligendamm". In: Republikanischer Anwältinnen- und Anwälteverein e. V. (Hrsg.): Feindbild Demonstrant. Polizeigewalt, Militäreinsatz, Medienmanipulation. Der G8-Gipfel aus Sicht des Anwaltichen Notdienstes. Berlin/Hamburg: 111-131.

Bröckling, Ulrich (2008): "Vorbeugen ist besser... Zur Soziologie der Prävention". Behemoth. A Journal on Civilisation 1/2008: 38-48.

Burke, Kenneth (1969a): A Grammar of Motives. Berkeley: University of California Press.

Burke, Kenneth (1969b): A Rhetoric of Motives. Berkeley: University of California Press.

Busse, Dietrich (2013): "Frame-analytische Zugänge zu juristischer Semantik". Vortrag auf der Interdisziplinären Tagung Juristische Korpuspragmatik. Die Herausforderung des

\footnotetext{
15 Zum Verhältnis von Prävention und Risiko und den daran sich neu gestaltenden Aufgaben des Staates schreibt Knobloch (2008: 10): "Dass es besser ist, beliebige vorhersehbare Übel bereits im Vorfeld abzuwenden, und nicht erst dann, wenn sie selbst und ihre manifesten Folgen eingetreten sind, ist ohne weiteres evident. Das verschafft einer Präventionsrhetorik von vornherein einen Vorteil gegenüber allen 'nachträglichen' Korrekturen und Kompensationen. Hier verhalten sich Gesundheit, Jugendkriminalität, Bildung, Terrorismus ganz analog. Das ist die Alltagsevidenz. Die politische Wirkung ist eine ganz andere. Unter dem Schirm der 'Prävention' verschiebt sich die Tätigkeit des Staates von der Durchsetzung der Gesetze hin zum Management von 'Risiken"'.
} 
Rechts durch Sprach- und Medientheorie. Freiburg Institute for Advanced Studies (FRIAS). Freiburg, 26.04.2013.

Fraenkel, Ernst (2012): Der Doppelstaat. Leipzig: CEP Europäische Verlagsanstalt Leipzig. Galli, Matteo/Preusser, Heinz-Peter/Franke-Penski, Udo (Hrsg.) (2006): Mythos Terrorismus. Vom Deutschen Herbst zum 11. September. Heidelberg: Winter.

Grunwald, Henning/Pfister, Manfred (2007): Krisis! Krisenszenarien, Diagnosen und Diskursstrategien. München: Fink.

Habscheid, Stephan/Koch, Lars (2014): "Katastrophen, Krisen, Störungen. Einleitung". In: Koch, Lars/Habscheid, Stephan (Hrsg.): Katastrophen, Krisen, Störungen. LiLi Zeitschrift für Literaturwissenschaft und Linguistik 173. www.lili.uni-siegen.de/ ausgaben/2014/lili173.html?lang=de [22.09.2015].

Holzinger, Markus/May, Stefan/Pohler, Wiebke (2010): Weltrisikogesellschaft im Ausnahmezustand. Weilerswist: Velbrück Wissenschaft.

Jäger, Margarete/Jäger, Siegfried (2007): Deutungskämpfe. Theorie und Praxis kritischer Diskursanalyse. Wiesbaden: VS Verlag für Sozialwissenschaften.

Knobloch, Clemens (2008): Der Präventivstaat und seine Feinde. Philipps-Universität Marburg. Marburg, 19.05.2008. Vorlesungsskript: www.online.uni-marburg.de/isem/sose08/ docs/praeventiv.pdf [14.09.2015].

Link, Jürgen (1992): "Normalismus: Konturen eines Konzepts". kultuRRevolution 27: 50-70.

Link, Jürgen (2009): Versuch über den Normalismus. Wie Normalität produziert wird. 2. Auflage. Opladen/Wiesbaden: Westdeutscher Verlag.

Müller, Jutta; Schmitz, Walter (Hrsg.) (2010): "Hoffentlich wird es nicht so schlimm, wie es ist." Zu Ursachen, Auswirkungen und Wahrnehmungen der "Krise". Dresden: Thelem.

Overington, Michael A. (1977): "Kenneth Burke and the Method of Dramatism". Theory and Society 4: 131-156.

Parr, Rolf (2009): "Kollektivsymboliken der Finanzkrise(n)". In: DISS Journal 18. www.dissduisburg.de/2009/12/kollektivsymboliken-der-finanzkrisen/ [20.02.2013].

Parr, Rolf (2010, i. E.): "Nach der Krise ist vor der Krise. Symbole und Narrative der Stadienabfolge in der Börsenberichterstattung von Print- und AV-Medien". In: Müller, Jutta/Schmitz, Walter (Hrsg.): "Hoffentlich wird es nicht so schlimm, wie es ist." Zu Ursachen, Auswirkungen und Wahrnehmungen der "Krise". Dresden: Thelem.

Rucht, Dieter/Teune, Simon (Hrsg.) (2008): Nur Clowns und Chaoten? Die G8-Proteste in Heiligendamm im Spiegel der Massenmedien. Frankfurt a. M.: Campus.

Rühle, Alex/Brendecke, Arndt (2011): "Action! Sonst Untergang! Der Historiker Arndt Brendecke über Krisenrhetorik". In: Süddeutsche Zeitung 03.12.2011.

Schmitt, Carl (1932/1996a): Der Begriff des Politischen. Text von 1932 mit einem Vorwort und drei Corollarien. 6. Auflage. Berlin: Duncker/Humblot.

Selz, Christian (2008): "Der Freunde Helfer?" über Agenturberichterstattung zum G8-Gipfel 2007 in Heiligendamm. Unveröffentlichte Diplomarbeit.

Spindler, Susanne/Tonks, Iris (Hrsg.) (2007): AusnahmeZustände. Krise und Zukunft der Demokratie. Münster: Unrast.

Ullmann, Karen (2007): "Das Ampelsystem. Polizeiliche Gefahrenprognosen während des Gipfels." In: Republikanischer Anwältinnen- und Anwälteverein e. V. (Hrsg.): Feindbild 
Legitimierung von sonder- und außerrechtlichen Maßnahmen

Demonstrant. Polizeigewalt, Militäreinsatz, Medienmanipulation. Der G8-Gipfel aus Sicht des Anwaltichen Notdienstes. Berlin/Hamburg, Assoziation A: 33-43.

Vogel, Friedemann (2010): "Blinde Flecken in der juristischen Hermeneutik. Zur mangelnden Lesbarkeit des Rechts". In: Rechtstheorie 41: 25-33.

Vogel, Friedemann (2012): Linguistik rechtlicher Normgenese. Berlin: de Gruyter.

\section{Korpusverzeichnis ${ }^{16}$}

BAO Kavala (2007): Kavala Report 1. Hrsg. v. Landesamt für innere Verwaltung. Landespolizei Mecklenburg-Vorpommern (1/2007).

Boehme, Christian (2012): Geplante Einschränkung der Reisefreiheit. In: www.theeuropean.de [26.04.2012].

Bundesamt für Bevölkerungsschutz und Katastrophenhilfe (2005): Das Bundesamt für Bevölkerungsschutz und Katastrophenhilfe. Selbstdarstellung auf der Homepage. Online verfügbar unter www.bbk.bund.de/DE/DasBBK/UeberdasBBK/ueberdasbbk_node.html [15.08.2012].

Council of the European Union (2004): Security handbook for the use of police authorities and services at international events. Brüssel, Arbeitsgrundlage.

Landtag Mecklenburg-Vorpommern (2011): Gesetz über die öffentliche Sicherheit und Ordnung in Mecklenburg-Vorpommern - Sicherheits- und Ordnungsgesetz MecklenburgVorpommern. SoG M.-V., in der Fassung der Bekanntmachung vom 09.05. (GVOB1.M.V. S. 176), seit dem 31.03.2011 geltende Fassung.

Schier, Mike/Holzapfel, Matthias/Schnürer, Christof (2014): "Der G8-Gipfel 2015". MerkurOnline. www.merkur-online.de/lokales/garmisch-partenkirchen/landkreis/g8-gipfel-2015schloss-elmau-3328475.html [13.06.2014].

Zeit Online. BKA führt eine Million Bürger als Drogenkonsumenten. www.zeit.de/politik/deutschland/2014-09/bundeskriminalamt-daten-buerger-straftaeter [24.09.2014].

13 IM OBR VS (24.04.2006): Erste Vorstellung des Konzeptes zum Umgang mit der Presse anlässlich G8. Zentralarchiv der Landespolizei Mecklenburg-Vorpommern in Schwerin, 200.14.13 519. Interne Anweisung zum Umgang mit Medien.

205 PM MdI M.-V. (28.12.2006): Hotel in Heiligendamm mit Farbe beworfen. Schwerin.

20 RB MdI M.-V. (11.01.2007): Rechtliche Bewertung der Einreise und Aufenthalte von ausländischen Störern. Zentralarchiv der Landespolizei Mecklenburg-Vorpommern in Schwerin, 200.14.13 $600 \mathrm{~B}$.

2 IM Kavala SHB (16.01.2007): Regelungen des Landesmeldegesetzes zu vereinfachten Auskünften. Zentralarchiv der Landespolizei Mecklenburg-Vorpommern in Schwerin, 200.14.13 598A. Interne Mittelung der Leitung der BAO Kavala an "B8 -> alle Nutzer".

206 PM MdI M.-V. (17.01.2007): Grußwort des Innenministers von M.-V. Lorenz Caffier.

Anlässlich der Indienststellung des Landeskommandos M.- V. in Schwerin am 17.01.2007. Schwerin.

28 IM BMI VS (27.04.2007): Anweisungen zu Grenzkontrollen. Zentralarchiv der Landespolizei Mecklenburg-Vorpommern in Schwerin, 200.14.13 591 C.

\footnotetext{
${ }^{16}$ Die Verzeichnung des Korpus erfolgt nach einem Archivierungs- und Nummerierungssystem der Autorin.
} 
207 PM MdI M.-V. (22.05.2007): Innenminister Caffier stellt Verfassungsschutzbericht 2006 vor. Schwerin.

27 MA Kavala EA3 (12.06.2007): Analyse der Medienberichterstattung zum G8-Gipfel. Zentralarchiv der Landespolizei Mecklenburg-Vorpommern in Schwerin, 200.14.13 388.

Innenministerium Mecklenburg-Vorpommern (13.06.07): Pressemitteilung. Schwerin.

GdP (20.06.2007): Polizisten aus dem ganzen Bundesgebiet kritisieren G8-Einsatz. Pressemitteilung.

OLG Rostock: Mitteilung vom 28.08.2007, Aktenzeichen 3 W 109/07.

BMI (08.10.2013): Schengen-Governance-Mechanismus beschlossen. Pressemitteilung. 